\title{
ESTUDIOS
}

\section{Valores y tiempo libre. Desafíos éticos de las redes sociales a la educación moral'}

\section{Agustín Domingo Moratalla²}

Resumen: El objetivo de estas páginas es analizar la relación entre la educación en valores y las organizaciones juveniles que promueven una educación democrática, también llamadas organizaciones de tiempo libre. El análisis describe los desafíos éticos que las TIC y las redes sociales plantean en el ámbito de la educación democrática como determinación de la educación moral. Análisis que se plantea como ejercicio de ética aplicada para promover la deliberación moral y la investigación interdisciplinar en los ámbitos socio-educativos. Utilizaremos la hermenéutica crítica como método que contribuye a la clarificación de las categorías éticas que intervienen en los proyectos de vida moral. La hipótesis del trabajo es sencilla: si las redes sociales están transformando las prácticas educativas, ¿̇en qué medida exigen una revisión y transformación de las categorías éticas tradicionales y una corresponsabilización de los agentes educativos?

Palabras clave: Educación democrática, Ética aplicada, ciudadanía, redes sociales y TIC, organizaciones juveniles, familia, mediación.

Fecha de recepción: 9 de mayo de 2011 .

Fecha de admisión definitiva: I5 de marzo de 2012.

\footnotetext{
' Este estudio se inserta en el Proyecto de Investigación Científica y Desarrollo Tecnológico FFI2010-21639 -C02-01, financiado por el Ministerio de Ciencia e Innovación y con Fondos FEDER de la Unión Europea, y en las actividades del grupo de investigación de excelencia PROMETEO/2009/085 de la Generalitat Valenciana.

2 Universidad de Valencia.
} 


\section{Leisure and values. Ethical cha- llenges of social networks on moral education}

\begin{abstract}
This paper discusses the relationship between imparting values and those youth organizations that promote democratic education, also called leisure organizations. The analysis describes the ethical challenges posed by ICTs and social networks within the framework of democratic education as a promoter of moral education. This analysis is proposed as an exercise in applied ethics to promote moral reflection and interdisciplinary research in the socio-educational scenario. We will use critical hermeneutics as a method to contribute to the clarification of those ethical categories that intervene in projects involving morality. The working hypothesis is simple: if social networks are transforming educational practices, to what extent do they call for a review and transformation of traditional ethical categories and the co-responsibility of educational agents?
\end{abstract}

Key Words: Democratic education, applied ethics, citizenship, social networks and ICTs, youth organizations, family, mediation.

\section{Valeurs et temps libre. Défis éthiques des réseaux sociaux à l'éducation morale}

Résumé: L'objectif de ces pages est d'analyser la relation entre l'éducation dans les valeurs et les organisations juvéniles qui promeuvent une éducation démocratique, également appelées organisations de temps libre (loisirs). L'analyse décrit les défis éthiques que les TICs et les réseaux sociaux posent dans le cadre de l'éducation démocratique comme détermination de l'éducation morale. Cette analyse est présentée comme un exercice d'éthique appliquée afin de promouvoir la réflexion morale et la recherche interdisciplinaire dans le cadre socio-éducatif. Nous utiliserons I'herméneutique critique comme méthode qui contribue à la clarification des catégories morales qui interviennent dans les projets d'une vie morale. L'hypothèse de travail est simple: si les réseaux sociaux sont en train de modifier les pratiques éducatives, dans quelle mesure exigent-ils une révision et une transformation des catégories éthiques traditionnelles et une co-responsabilisation des agents éducatifs?

Mots clé: Éducation démocratique, éthique appliquée, citoyenneté, réseaux sociaux etTIC, organisations juvéniles, famille, médiation.

\section{Introducción}

El objetivo de estas páginas es analizar la relación entre la educación en valores y las organizaciones juveniles que promueven una educación democrática, también llamadas organizaciones de tiempo libre. El análisis describe los desafíos éticos que las TICs y las redes sociales plantean en el ámbito de la educación democrática como determinación de la educación moral. Análisis que se plantea como 
ejercicio de ética aplicada para promover la deliberación moral y la investigación interdisciplinar en los ámbitossocio-educativos. Utilizaremos la hermenéutica crítica como método que contribuye a la clarificación de las categorías éticas que intervienen en los proyectos de vida moral. La hipótesis del trabajo es sencilla: si las redes sociales están transformando las prácticas educativas, ¿̇en qué medida exigen una revisión y transformación de las categorías éticas tradicionales y una corresponsabilización de los agentes educativos?

Respondemos a esta pregunta en tres momentos. En primer lugar recuperando una visión integradora de la educación donde la escuela y la familia son completadas por un ámbito social definido como horizonte educativo del tercer maestro, con capacidad para integrar educación formal e informal. En segundo lugar proponiendo una revisión y transformación de la educación en valores como núcleo de las estrategias de educación democrática. Las redes sociales sitúan la ciudadanía democrática ante un desafío en el que tienen la oportunidad de transformarse en una ciudadanía digital o una ciudadanía democrática transformada. Las redes no suponen sólo un desafío técnico o instrumental sino una transformación del imaginario y, por consiguiente, exigen una revisión, actualización y transformación de las categorías con las que, hasta ahora, habíamos planteado la educación democrática. La educación en valores se enriquece así con la actualización de categorías básicas como las de virtud, actitud o carácter. En tercer lugar se describen algunos retos de esta transformación para el trabajo de las organizaciones juveniles de tiempo libre como agentes para una educación democrática integral.

\section{2. Ética aplicada y tiempo libre}

\section{I. Ética aplicada y redes sociales}

Las redes sociales están planteando numerosos desafíos a la educación moral ${ }^{3}$. De la misma forma que los planteamientos alarmistas o apocalípticos son poco productivos, tampoco los planteamientos románticos o acríticos son útiles para quienes tienen responsabilidades educativas, bien como padres o bien como

\footnotetext{
3 Usaremos el término de "redes sociales" en un sentido amplio para referirme no sólo al uso de Internet o la utilización de Facebook, Tuenti, Messenger o los diferentes tipos de "bloging". Describo el conjunto de múltiples dispositivos propios de las tecnologías de la información y la comunicación, desde el uso del celular hasta el "bloging" pasando por otros procesos web o dispositivos multimedia.
} 
maestros. En palabras de Ch. Sádaba, una de las mejores conocedoras de este nuevo entorno educativo, hay nuevos riesgos que derivan del alcance o magnitud de estas herramientas (exposición de la intimidad), de la intensidad y duración de los daños (acoso al diferente) y de las formas en las que se produce la socialización (adscripción a grupos). Si antes la intimidad se exhibía a 15 ○ 20 personas, ahora está disponible para cientos y miles de semejantes. Si antes había situaciones de acoso en el colegio o la calle, ahora el acoso se produce en todos los ámbitos y tiempos en los que los menores están conectados. A pesar de lo que muchos piensen, entre los riesgos no está el aislamiento, sino el aislamiento de los padres y de los profesores, no de los compañeros o pares. Las investigaciones derrumban el mito de que las redes llevan al aislamiento y plantean nuevos desafíos que deben ser afrontados evitando simplificaciones. ${ }^{4}$

Desde la ética aplicada no podemos permanecer indiferentes ante estos desafíos para revisar la actualidad de categorías e instituciones que hasta ahora habían sido útiles para la educación moral ${ }^{5}$. Es el caso del protagonismo de los valores o de instituciones como la familia y la escuela con las que, hasta ahora, habíamos planteado el horizonte de la educación moral en una sociedad democrática. Las redes sociales nos abren un horizonte nuevo y tenemos que preguntarnos ¿cómo actualizar estas categorías e instituciones?, ¿̇sigue siendo válida una educación en valores o tenemos que proponer otras categorías como las virtudes o actitudes?, ¿qué papel desempeñan las redes sociales y el tiempo libre en la configuración de nuevas vías educativas? En este contexto social y educativo de interactividad podemos llegar a preguntarnos żqué papel desempeña la escolarización en las nuevas formas de educación democrática como educación para la ciudadanía activa? No es fácil responder a esta pregunta pero es quizá la primera que deberíamos plantearnos cuando nos preguntamos por el valor educativo de las actividades que se realizan en un tiempo que imprecisamente se llama "libre".

Para responder a estas preguntas podemos empezar clarificando qué entendemos por tiempo libre. Desde un enfoque utilitarista e incluso pragmático, tiempo

${ }^{4}$ Cfr. Ch. SÁdABA, "Los jóvenes e Internet", Entrevista en XL Semanal, 21-27/VIII/2011. Autora del libro (2011) Menores y Redes sociales, Barcelona, Fundación Telefónica-Ariel.

${ }^{5}$ Por ética aplicada entendemos el ámbito de la filosofía moral que clarifica, fundamenta y discierne para realizar las categorías morales básicas de la acción e interacción humana (bienes, valores, virtudes, deberes, derechos). Hemos desarrollado este concepto en nuestros trabajos (2008) Ética para Educadores, Madrid, PPC; (2009) "El desencantamiento de la ética aplicada. Atreverse a pensar nada menos que una ética sin más", en A. CAÑón y A. VILLAR (ed.) Ética pensada y compartida. Homenaje a A. Hortal. Madrid, UPCO, pp. 51-71. 
libre es un concepto impreciso e indeterminado: a diferencia del tiempo del trabajo o del tiempo de los negocios, el tiempo libre se presenta sin valor alguno; a diferencia del tiempo del trabajo, de formación o de ocupación, es un tiempo de entretenimiento, de ocio $y$, aparentemente, sin valor formativo. Como no está fuera de la acción humana y el conjunto de actuaciones que configuran la identidad de las personas, acaba siendo un tiempo importante que puede llenarse de actividades 6 . Surgen actividades de tiempo libre, incluso organizaciones que gestionan y administran el tiempo libre. Desde este enfoque, algunas organizaciones juveniles que no se desentienden de la educación democrática o formación integral, se presentan como organizaciones para mantener a los jóvenes activos, entretenidos y, sobre todo, para evitar la desocupación como distracción.

\subsection{Educación democrática y tiempo libre}

Sin analizar las posibilidades que el concepto aristotélico y de vida activa tendría en estos planteamientos, aparecen así dos tipos de tiempo: productivo y libre ${ }^{7}$. Aplicado al ámbito educativo nos encontramos que el primer tipo de tiempo corresponde al tiempo de la escolarización o educación formal y el segundo al tiempo de ocio donde incluiríamos la educación no formal. Aquí comienzan los problemas de la educación democrática porque algunos teóricos de la educación reducen sus análisis al problema de la escolarización. Esto no significa que no tengan en cuenta el papel de la televisión, los videojuegos, la calle o las bibliotecas en la educación democrática, significa que quizá les asignan un papel accidental, marginal y secundario. Las investigaciones sobre educación democrática no siempre han prestado la atención necesaria al ámbito de la educación informal.

Por la importancia que ha tenido entre los teóricos de tradición post-rawlsiana, los trabajos de Amy Gutmann muestran si no la marginación, sí el olvido del valor que tiene la educación fuera de las aulas para la construcción de una ciudadanía

\footnotetext{
"La distinción entre "tiempo de trabajo" y "tiempo libre" tiene un valor instrumental en la filosofía social moderna y es fácilmente cuestionable cuando se adoptan horizontes filosóficos más amplios de tradición aristotélica, personalista o hermenéutica. Sin necesidad de remontarnos a las tesis de Huizinga y Geertz sobre el valor del juego como elemento unificador del tiempo humano, las consecuencias éticas de esta separación instrumental y utilitaria ha sido descritas por R. SENNET en sus trabajos: (2001) La corrosión del carácter, Barcelona, Anagrama; (2006) La cultura del nuevo capitalismo, Barcelona, Anagrama.
}

${ }^{7}$ Esta tradición aristotélica, ciceroniana y republicana (entendido este término en sentido amplio) ha sido retomada en la filosofía política por Hannah ARENDT en su trabajo (2005) La condición humana, Barcelona, Paidós. 
democrática. En su clásico trabajo sobre educación democrática sólo dedica un capítulo a lo que llama educación fuera de las aulas y en él plantea de una forma rápida la contribución de las bibliotecas, las televisiones y las nuevas tecnologías $^{8}$. Aunque en su planteamiento de las nuevas tecnologías se está refiriendo al vídeo y la televisión por cable, es consciente del espacio educativo que dejamos fuera de la familia y la escuela:

Entre la autoridad pública que controla lo que se enseña a los niños en la escuela y la autoridad paterna que controla lo que se les enseña en el hogar se extiende "un gran territorio educativo" ocupado por bibliotecas, tiendas de libros, museos, periódicos, cines, radio, televisión y otras instituciones culturales cuyos títulos de propiedad no son tan obvios. Sin embargo, si es obvio que las decisiones independientes de los padres no pueden determinar la existencia de estas instituciones o si estas sirven para educar a los niños. Los padres pueden permitir o prevenir, alentar o desalentar a sus hijos para ir a bibliotecas o ver televisión educativa, pero no pueden proporcionar el acceso a cualquiera de estas fuentes si estas no existen por virtud de una decisión política. Sin embargo, lo bien educados que resulten los niños fuera de la escuela depende en gran medida del acceso que tengan a tales fuentes, que se encuentran más allá del poder individual de los padres. ${ }^{9}$

Si la educación democrática se limita a la familia y las aulas, entonces dejamos fuera lo que Guttman llama un gran territorio educativo. Si añadimos las investigaciones de algunos sociólogos de la educación cuando nos recuerdan que lo importante de la vida ocurría fuera de clase, entonces va cobrando sentido la necesidad de plantearnos el protagonismo progresivo de otras instancias educativas que no son las aulas o las familias ${ }^{10}$. Partiendo de estos desafíos ha surgido

${ }^{8}$ En el conjunto de las teorías contemporáneas de la educación, las reflexiones sobre la "educación democrática" se han planteado desde los diferentes paradigmas de "educación moral". Un marco valioso para situar estas aportaciones ha sido descrito por R. M BuXARRAIS en su trabajo (2000) "Tendencias y modelos de educación moral": Diálogo Filosófico, 47, pp. 196-220 y por V. Gozálvez (2010) "Hacia una reconstrucción de la razón pedagógica": Teoría de la Educación, 22:2, pp. 19-42.

9 A. Guttman (2001) La educación democrática, Barcelona, Paidós, p. 288. Subrayado nuestro. El ritmo de dos cambios producidos en el ámbito de las TIC han sido mucho más acelerado que el de los cambios producidos en el ámbito de la filosofía políitica. Precisamente, cuando los planteamientos post-rawlsianos han intentado aplicarse al ámbito de educativo se han planteado con mayor intensidad los límites de la escuela y la educación formal en los aprendizajes democráticos. En el ámbito de la teoría política son clásicos los trabajos de G. SARTORI (1998) La sociedad teledirigida, Madrid, Taurus; (2003) Videopolítica, Madrid, FCE; y C. R. SunsteIn (2003) República.com, Barcelona, Paidós. En el ámbito pedagógico es interesante la actualización del debate que realizan R. GARCíA y V. GozÁlvez (2010) Repensando la educación: cuestiones y debates para el siglo XXI, Valencia, Brief.

${ }^{10}$ Cfr. F. Dubet, La Vanguardia Magazine, 19/09/2010. En el ámbito la educación informal es importante el reciente trabajo de S. ROMI y M. SCHMIDA (2009) "Non-formal education: a major educational force in the postmodern era": Cambrigde Journal of Education, 39:2, pp. 257-273. 
un amplio campo de investigación en ciudadanía activa ("activ citizenship") y aprendizaje a lo largo de la vida en las sociedades del conocimiento ("learning society"). ${ }^{11}$

Este gran territorio educativo resulta especialmente conflictivo y problemático en el análisis de la adolescencia como etapa de transición entre la infancia y la juventud. Entre nosotros, el reciente informe de la Fundación Encuentro sobre los adolescentes en la ESO constata que nos hallamos ante una adolescencia nueva y distinta, incluso plantea la necesidad de un nuevo paradigma pedagógico consensuado. Dos datos son importantes: (a) nos hallamos ante una adolescencia nueva y distinta, (b) se ha transformado el aprendizaje en esta etapa porque hay nuevas fuentes de información y nuevos magisterios (subrayado nuestro). Según el informe, los adolescentes de hoy:

no son más maduros que los adolescentes de otras épocas, pero sí experimentan y expresan anticipadamente conductas que, hasta no hace mucho tiempo, eran habituales de la juventud, pero no de la adolescencia. Se da, pues, en ellos una contradicción o disyunción entre maduración psicológica personal y determinados rasgos psicosociales adelantados. La frontera entre la infancia y la edad adulta parece difuminarse. Este dato contrasta paradójicamente con la larga permanencia de las jóvenes generaciones en el hogar familiar y su tardanza en emanciparse. Por otro lado, resulta innecesario decir que, al menos dentro de la cultura occidental... los adolescentes de un país son iguales a los de otros, con miles de kilómetros de distancia por medio... ${ }^{12}$

Esta difuminación entre la infancia y la madurez, que se presenta aquí como cierto adelanto de la madurez en la adolescencia por determinadas conductas, es presentada por otros investigadores como una adolescentización de la juventud. Un hecho que requiere una especial consideración en las estrategias formativas de esta edad intermedia porque está generando una adolescentización de toda la sociedad española ${ }^{13}$.

${ }^{11}$ Cfr. R. BENN (2000) "The genesis of active citizenship in the learning society": Studies in the Education of Adults, 2:32, pp. 241-256; Z. MENDIWELSO et alii (2006) Takepart learning framework for activ learning for active citizenship, London, Home Office. Hemos desarrollado estos planteamientos en recientes trabajos: (2006) Ciudadanía, religión y educación moral, Madrid, PPC; (2011) Ciudadanía activa y religión, Madrid, Encuentro. Hemos aplicado este planteamiento en el libro (2006) Formación para la Convivencia, Valencia, Observatorio para la Convivencia escolar.

12 Fundación EnCUENTro (2010) Informe España 2010, Madrid, p. 109.

${ }^{13} \mathrm{~J}$. Elzo (2008) La voz de los adolescentes. Madrid, PPC, p. 7. 


\subsection{El horizonte del tercer maestro y las nuevas generaciones}

Todos los estudios destacan que el papel que la revolución tecnológica y el conjunto de las TIC (Tecnologías de la Información y el Conocimiento) están desempeñando en los procesos madurativos de esta edad, y de manera especial lo que con cierta imprecisión se ha llamado redes sociales. Nos encontramos ante nuevas generaciones de menores que han crecido en un ambiente nuevo y que ha sido calificada como generación "interactiva", "celular", "multifuncional" y "multitarea"14. Nuevas generaciones que plantean nuevos retos educativos, unas veces como riesgos para el desarrollo de la personalidad del menor y otras como desafíos para padres, maestros y líderes sociales con responsabilidades formativa.

Ante el uso anónimo e incontrolado de las redes sociales, los redactores de la Fundación Encuentro reclaman un nuevo paradigma comprensivo para entender a los menores y adolescentes de hoy. Incluso piden cambios radicales en los aprendizajes que se producen en la adolescencia, sobre todo en lo que se ha llamado la Enseñanza Secundaria Obligatoria (ESO). Cambios que reclaman porque constatan no sólo la impotencia de la familia y la escuela en el compromiso educativo, sino la incapacidad de estos magisterios tradicionales para gestionar los nuevos conocimientos. En palabras de Elzo: "Es la generación que más sola está creciendo, produciéndose así una autoafirmación a través básicamente del grupo de amigos y los diferentes medios de comunicación, con Internet cada vez más a la cabeza..."15.

Estas transformaciones deberían llevarnos a imaginar nuevas formas de relación entre la educación formal y la no-formal, entre la calle y la escuela, entre las redes sociales y los hábitos escolares. Deberíamos preguntarnos si las transformaciones que se están produciendo en la adolescencia con el protagonismo relevante de las redes sociales no nos tendrían que llevar a proponer una transformación de las instancias educativas tradicionales, incluso distintas al magisterio de la familia y de la escuela. Necesitamos repensarlas desde un horizonte educativo nuevo y radical que podemos llamar el horizonte del tercer maestro. Podríamos describir este nuevo magisterio con la expresión Tercer maestro y preguntarnos cómo per-

\footnotetext{
${ }^{14}$ Estos calificativos han sido los utilizados por X. Bringué, Ch. Sádaba y J. Tolsá como investigadores de la Fundación Telefónica para presentar el informe La generación interactiva en Iberoamérica 2010, Madrid, 2011. Véase sobre todo, pp 541 y ss.

15 J. Elzo, op. cit., p. 9.
} 
filarlo desde las actividades que se realizan en un tiempo que no por el hecho de ser libre tiene que dejar de ser educativo.

Si en los planteamientos del primer magisterio de la familia y el segundo magisterio de la escuela, el tiempo libre se plantea como un tiempo educativamente indeterminado, quizá deberíamos empezar a plantearlo como un tiempo con un potencial educativo que aún está por explorar ${ }^{16}$. El potencial educativo del llamado tiempo libre puede ser útil para desarrollar esta hipótesis del Tercer maestro como horizonte educativo. De hecho, puede evitar una fractura que se ha detectado en los estudios sobre los adolescentes: la ruptura entre los valores finalistas y los valores instrumentales. Elzo utiliza esta distinción entre valores finalistas y valores instrumentales para describir la implicación distanciada respecto a los problemas y las causas que dicen defender:

los actuales jóvenes invierten afectiva y racionalmente en los valores finalistas (pacifismo, tolerancia, ecología, lealtad, etc.) a la par que presentan grandes fallas en los valores instrumentales sin los cuales todo lo anterior corre el riesgo de quedarse en un discurso bonito. Me refiero a los déficits que presentan en valores tales como el esfuerzo, la autorresponsabilidad, el compromiso, la participación, la abnegación (que no sabe lo que es), el trabajo bien hecho, etc. De ahí que la tolerancia y la solidaridad queda más en mera querencia, más en un deseo que en una realización concreta si no va acompañada de valores instrumentales ${ }^{17}$.

\section{Educación en valores y ciudadanía digital}

\section{I. De los valores a las virtudes}

Esta distinción entre valores finalistas y valores instrumentales es útil para conocer los límites intrínsecos de la educación en valores como uno de los paradigmas prevalentes de educación democrática. Es útil por dos razones: en primer lugar porque nos recuerda la diferencia entre fines y medios, entre grandes metas y pequeños hábitos, entre la moral pensada y la moral vivida. En segundo lugar,

\footnotetext{
${ }^{16}$ Hemos realizado algunos trabajos en esta dirección, cfr. (2003) Calidad Educativa y Justicia social, Madrid, PPC. Para algunos investigadores, la aceleración de los cambios y la disolución de las fronteras entre lo formal y lo informal están exigiendo replantear el aprendizaje como "des-aprendizaje", cfr. E. MCWILIAM (2008) "Unlearning how to teach": Innovations in Education and Teaching International 45:3, pp. 263-279.

17 J. Elzo, op. cit., p. 15.
} 
porque exige clarificar educativamente el concepto de valor, su jerarquización en la gestión educativa y, sobre todo, sus límites reales para forjar el carácter de los jóvenes o promover el sentido de una comunidad justa. El éxito que ha tenido el concepto de valor en la organización de los sistemas educativos no justifica el olvido de otras categorías educativas importantes como las de virtud, carácter, actitud o incluso ejemplaridad ${ }^{18}$.

Cuando analizamos el potencial educativo del tiempo libre y el protagonismo que las redes sociales tienen en la configuración de la personalidad juvenil descubrimos la necesidad de completar los valores con otras categorías básicas de educación moral como temperamento, virtudes, carácter y actitudes ${ }^{19}$. Las organizaciones juveniles del tiempo libre (escultismo, juniors y otras asociaciones juveniles) no pueden limitarse únicamente a establecer programas de educación en valores, están obligadas a recuperar elementos vinculados a otras formas no cognitivas de entender los aprendizajes, es decir, otras formas no intelectivas sino emocionales, cordiales y actitudinales de organizar la educación moral. Los responsables de la educación no formal deben plantearse qué entienden por virtud, qué tipos de virtudes quieren promover, qué tipo de caracteres incentivan $y$, sobre todo, qué tipo de actividades son las más adecuadas para forjar determinadas actitudes, temperamentos y virtudes en los jóvenes.

Es lógico que las organizaciones juveniles limiten su discurso organizativo a la educación en valores porque de esa forma alinean sus estrategias formativas con las que, hasta ahora, se planteaban en el horizonte de la ciudadanía democrática. Los valores de la sociedad europea de la postguerra fueron integrados en las constituciones y por eso los diferentes sistemas educativos han apelado a ellos

${ }^{18}$ Cfr. J. SÁez CRUz (2005) Educar en la virtud y la virtud de educar. Educar para la Justicia, Burgos, Instituto Superior de Filosofía San Juan Bosco. El cuestionamiento de los "valores" como categoría prioritaria y su desplazamiento por otras categorías más clásicas como las de "actitud", "virtud" o "carácter" están siendo reforzadas por las aportaciones de la neuroética al campo de la educación, en este sentido cfr. K. SherIDAN, E. ZinCheNKO y H. GARDNeR, "Neuroethics in Education", en Illes, I. (ed.) (2006) Neuroethics, Oxford University Press, pp. 265-275. Con interés para el mundo de la educación infantil, cfr. J. A. MARINA (2011) El cerebro infantil: la gran oportunidad, Barcelona, Ariel, sobre todo p. $67 \mathrm{ss}$.

${ }^{19}$ Estas categorías son básicas y fundamentales en cualquier teoría ética. La recuperación que hace Maclntyre de la categoría de virtud ((1989) Tras la virtud, Barcelona, Crítica) puede ser utilizada para la reconstrucción neoaristotélica de las categorías educativas pero también para la reconstrucción personalista y comunitaria de las diferentes tradiciones pedagógicas. Para una clarificación de estos conceptos puede verse nuestro trabajo (2008) Ética para Educadores, Madrid, PPC. 
para coordinar las metas de la escuela, la familia y el conjunto de la sociedad 20 . Ahora bien, este potencial normativo de los valores para construir una ciudadanía democrática no exige el olvido o la marginación del potencial educativo de las virtudes, el carácter o las actitudes para promover una formación integral de la persona.

El horizonte educativo del tercer maestro puede recuperar el potencial educativo de conceptos tradicionales como virtud, carácter o actitud con los que completar el potencial normativo de los valores. Las insuficiencias de la familia y la escuela en la educación juvenil se hacen más explícitas a medida que limitamos nuestro análisis a la educación en valores. La modernización de los procesos educativos ha exigido referencias éticas que sean plausibles para sociedades plurales, por eso la educación en valores ha permitido el reconocimiento de unos mínimos éticos comunes. Ahora bien, la modernización no es un proceso de homogeneización o de simplificación de parámetros educativos. El reconocimiento de una ética mínima para la promoción de una ciudadanía democrática (educación en valores) no excluye la pluralidad de fuentes, tradiciones educativas y de propuestas morales. El horizonte del tercer maestro permite reconstruir las teorías de la ciudadanía democrática desde una ética hermenéutica que incorpora el horizonte de los contextos sociales, las tradiciones familiares y las narrativas comunitarias ${ }^{21}$. De esta forma no perdemos el horizonte cognitivo y post-convencional de una educación democrática pero lo enriquecemos y enraizamos dentro de horizontes narrativos que no son sólo formales o institucionales sino informales y culturales ${ }^{22}$.

\footnotetext{
${ }^{20}$ Hemos analizado la relación entre familia y educación democrática en nuestros trabajos: (2006) Ética de la vida familiar. Claves para una ciudadanía democrática, Bilbao, Desclée; "Familia y ciudadanía activa. El capital social familiar en el espacio público educativo" en M. T. López (editora) (2007) Familia, Escuela, Educación, Madrid, Cinca-Fundación Acción Familiar, pp. 53-95.

${ }^{21}$ Sobre el concepto de ética hermenéutica, véase nuestro trabajo (2011) Ética. Una introducción, Madrid, Acento; Introducción a P. RICOEUR (1999) Lo justo I, Madrid, Caparrós; y P. RICOEUR (2009) Lo Justo II, Trotta, Madrid. Para una hermenéutica "crítica", cfr. J. Conill, Ética hermenéutica. Tecnos, Madrid, 2006.

$22 \mathrm{El}$ debate sobre la necesidad de tender un puente entre el horizonte postconvencional universalista y el horizonte histórico-contextual o narrativo está presente en los debates educativos recientes, cfr. P. ENSIIN, M. TJIATAS Y S. TODD (2009) "Philosophy of education and the gigantic affront of universalism": Journal of Philosophy of Education, 53:1, pp. 1-29. En ningún momento se trata de recuperar una "hegemonía del particularismo" sino recuperar el papel del educador como "traductor", en la línea de nuestras investigaciones sobre ética y hermenéutica, cfr. (1990) El arte de poder no tener razón. La hermenéutica dialógica de Gadamer, Salamanca, Publicaciones Univ. Pontificia Salamanca; Ciudadanía activa y religión, cap. 7, op. cit.
} 


\subsection{Redes sociales y nuevos agentes educativos}

Las redes son un ámbito estratégico para la referencia educativa de maestros y padres. Incluso se ha llegado a plantear la necesidad de una "familia interactiva" que integre a padres e hijos en una nueva dimensión de las TICs. Son todavía pocos los padres implicados en la mediación familiar ante las pantallas perdiendo la oportunidad de ser un referente educativo en este ámbito ${ }^{23}$. A padres y maestros nos han venido bien los valores porque nos han permitido unificar las categorías éticas de una ciudadanía democrática y mantenernos en un horizonte que, en términos cognitivos, podríamos llamar post-convencional que facilita el puente con los derechos humanos. Con ellos hemos descubierto la existencia de unos mínimos morales comunes, hemos clarificado su organización normativa y los hemos propuesto para orientar la convivencia en sociedades plurales. Ahora bien, debemos preguntarnos si el anunciado fracaso de la familia y la escuela en la educación de los adolescentes no está relacionado con la simplificación del horizonte educativo y el arrinconamiento de categorías como las de virtud, carácter, temperamento o actitud en los procesos de modernización educativa. Como padres y maestros no hemos querido perder el tren de la modernización educativa y por ello nos hemos identificado aceleradamente con el lenguaje universalista de los valores y derechos, como si la apelación a la virtud, la actitud o sencillamente la ejemplaridad, restara credibilidad y plausibilidad a nuestra propuesta de educación democrática.

Muchos padres y maestros han identificado la educación en valores como la única alternativa posible para la promoción de una ciudadanía democrática, como si desde otras tradiciones morales no fuera posible articular los procesos de modernización educativa con los valores de una sociedad pluralista y abierta. Esta simplificación no sólo ha incrementado la distancia entre la escuela la la que se le asigna un protagonismo especial en el espacio público) y la familia (que se restringe indebidamente como si sólo formara parte de espacio privados) sino que ha dejado el terreno abonado para que otros agentes como la televisión, la música, la noche, la calle $y$, especialmente, las redes sociales se conviertan en nuevos agentes educativos. De esta forma, aunque padres y maestros nos coordinemos para promover una ciudadanía democrática tradicional (educación en valores, educación para los derechos); las nuevas instancias educativas ponen en cuestión los agentes educativos tradicionales e invitan a trabajar en nuevos espacios públicos educativos, menos formalizados y más globalizados que exigen

${ }^{23}$ Sobre el "reto de las familias interactivas", cfr. X. BRINGUÉ, CH. SÁDABA y J. Tolsá (2011) La generación interactiva en lberoamérica, Madrid, Fundación Telefónica, pp. 555 ss. 
una ciudadanía democrática transformada que algunos han llamado ciudadanía digital. ${ }^{24}$

¿Qué tipo de magisterio están ejerciendo estas nuevas instancias educativas? ¿En qué medida la educación no formal o educación para el tiempo libre puede asumir los desafíos de este nuevo horizonte del tercer maestro? ¿̇Se trata de un desplazamiento de la responsabilidad a nuevos agentes o de la posibilidad de repensar sus funciones tradicionales? No es una tarea fácil y por ello es importante no despreciar tradiciones educativas que promueven la virtud, el carácter o la ejemplaridad en la gestión educativa. Las organizaciones juveniles no pueden limitarse a promover el sentido de pertenencia a una comunidad determinada o la participación en instituciones políticas tradicionales.

Las redes sociales reclaman una ciudadanía democrática transformada porque las estructuras de comunicación y relación intra e intergeneracionales se están modificando; el acceso a la información, la organización del conocimiento, los dinamismos de institucionalización y los procesos de liderazgo han sido modificados sustancialmente por las nuevas tecnologías. Como ya subrayó M. Castells, la sociedad de la información no sólo está introduciendo las redes como estructuras organizativas, sino como estructuras cognitivas, está modificando las identidades de los individuos y, por consiguiente, sus formas de pertenencia y participación social. ${ }^{25}$

Este impacto de las redes en los procesos tradicionales de identificación democrática ha llevado a una nueva ciudadanía digita ${ }^{26}$. No se trata de una versión postmoderna o deconstruida de la ciudadanía democrática sino la constatación de su necesaria transformación. Ahora bien, con el horizonte educativo del tercer maestro y la recuperación de otras categorías clásicas de educación moral no estamos proponiendo una retorno a categorías educativas premodernas sino la po-

\footnotetext{
${ }^{24}$ Sobre el concepto de ciudadanía digital puede verse nuestro trabajo (2009) "Infoética y Derechos humanos: posibilidades y límites de la ciudadanía digital": Revista de Fomento Social, 256:64, pp. 735-755.

${ }^{25}$ Cfr. M. CASTels (2001) La era de la información, Madrid, Alianza, vol. I. Estas transformaciones de la identidad han sido descritas con lucidez por Zygmunt Bauman. Una síntesis de sus propuestas puede verse en su libro (2010) El tiempo apremia. Conversaciones con C. Rovirosa-Madrazo, Barcelona, Arcadia.

${ }^{26}$ Para una clarificación de este concepto en el ámbito de la Infoética como ética aplicada puede verse nuestro trabajo (2009) "Infoética y derechos humanos. Posibilidades y límites de la ciudadanía digital": Revista de Fomento Social, 256:64, pp. 735-755.
} 
sibilidad de una crítica personalista y comunitaria de la educación democrática organizada únicamente en términos de valores. El horizonte educativo del tercer maestro es una oportunidad para reconstruir los programas tradicionales de ciudadanía democrática no sólo para adaptarlos a los nuevos entornos tecnológicos o instrumentales sino para revisar o transformar sus posibilidades y límites. Aquí es donde debemos averiguar si la ciudadanía digital es sólo una adaptación de la ciudadanía democrática a los entornos tecnológicos de las redes sociales o supone una transformación una transformación radical de lo que hasta ahora entendíamos por democracia o ciudadanía.

\section{Desafíos de las redes sociales a las organizaciones juveniles}

La posibilidad de revisar las estrategias educativas de ciudadanía democrática desde una ciudadanía digital no es un simple ejercicio de epistemología social sino un imperativo moral con dimensiones que afectan al diseño de las organizaciones juveniles del tiempo libre. Es un desafío de responsabilidad educativa que debemos afrontar desde diversos niveles y que exige un trabajo necesariamente interdisciplinar ${ }^{27}$. Algunos debates pedagógicos giran en torno a la autorización y uso de los teléfonos móviles en los tiempos o espacios educativos. Son debates en los que se ponen a prueba los valores de la educación en una sociedad democrática porque nos sentimos capacitados para pontificar sobre la tolerancia, la autonomía, la permisividad y la responsabilidad en los espacios públicos educativos. Son debates que se saldan habitualmente estableciendo reglas de juego claras entre los padres y los educadores, y que también se han producido en las organizaciones juveniles. Ahora bien, el espacio público educativo de las organizaciones juveniles tiene características diferentes. Mientras la obligatoriedad de la escolarización garantiza la plausibilidad formal y facilita la deliberación sobre un conjunto de reglas consensuadas, la voluntariedad e informalidad de las organizaciones juveniles incrementa la complejidad en la deliberación para el establecimiento de normas.

A esta complejidad organizativa debemos añadir la complejidad psicológica de los adolescentes $y$, sobre todo, la disparidad de criterios cuando gestionamos

\footnotetext{
${ }^{27}$ Sobre el concepto de interdisciplinariedad en la investigación social y su relación con los imperativos de responsabilidad ante los que nos sitúa el nuevo horizonte cultural puede verse nuestro trabajo: "Bioética y filosofía: dos versiones rivales de la interdisciplinariedad", en L. FeITO y T. DOMINGO (2011) Investigación en Bioética, Madrid, Dykinson, pp. 205-216.
} 
la responsabilidad juvenil. Disparidad que se transforma en divergencia y fácil confrontación ideológica cuando los educadores nos enfrentamos a la valoración de la responsabilidad penal de los menores. En las organizaciones juveniles se han incrementado las dificultades para establecer reglas de juego claras, no sólo porque los procesos de modernización educativa han producido una psicologización y hasta privatización de la moral juvenil sino porque los procesos de modernización social han generado una judicialización y hasta relativización de la responsabilidad de los menores. Estos procesos han afectado estructuralmente a unas organizaciones juveniles que no sólo se han tenido que adaptar a los nuevos cambios sociales y educativos sino a las incoherencias, discrepancias o arbitrariedades de la normativa que protege a los menores y regula su responsabilidad penal. ${ }^{28}$

Si al desconcierto generado por los cambios psicológicos y la emergencia de las redes sociales añadimos la complejidad autonómica a la que está sometida la regulación administrativa de las organizaciones juveniles, o la dispersión de criterios con los que jueces y fiscales administran referencias educativas tan importantes como las de daño moral, responsabilidad penal o madurez de un menor, no es de extrañar la crisis que que hoy vive el asociacionismo y la participación juvenil. A la complejidad de mantener una organización juvenil se añaden estos factores que dificultan la deliberación para establecer normas claras en la estructura y funcionamiento.

Esta complejidad de las organizaciones juveniles puede gestionarse mejor si delimitamos con precisión algunos desafíos educativos. Aunque los desafíos que voy a describir están directamente relacionados con el impacto de las redes sociales pueden ser útiles por tres razones bien diferentes. Primero para revisar los problemas de nuestra organización y elaborar un diagnóstico más certero. Segundo para clarificar el papel que nuestra organización ocupa en el conjunto de una sociedad civil que está transformando los imaginarios educativos. Tercero para orientar de una manera realista las actividades de la organización con la finalidad de facilitar la formación integral de los jóvenes y, si fuera posible, evitar el desánimo o la desmoralización de los educadores en contextos de ciudadanía digital.

\footnotetext{
${ }^{28}$ Hemos planteado estos temas en otro trabajo que lleva por título "Adolescencia y menor maduro. La autonomía personal como un desafío a la moral profesional", en M. DE LOS REYES y M. SÁNCHEZ (eds.) (2010) Bioética y Pediatría, Madrid, Sociedad de Pediatría de Madrid y Castilla la Mancha, pp. 48-57.
} 


\section{I. Evitar las simplificaciones y el reduccionismo}

Las redes sociales están ahí y cualquier estrategia educativa está obligada a contar con ellas. Están para todos y han venido para quedarse, por tanto la familia, la escuela y las organizaciones juveniles está obligadas a contar con ellas. Lo curioso de esta presencia de las redes sociales en estos espacios públicos educativos es la simplificación y el reduccionismo con los que suelen plantearse las deliberaciones. Una tendencia que deberíamos evitar despertando nuestra sensibilidad para evitar la simplificación de los apocalípticos, es decir, intérpretes pesimistas de los procesos culturales quienes consideran que la permisividad ante las redes sociales incrementa la vulnerabilidad de los adolescentes, el desconcierto educativo y la indefensión de los ciudadanos ante las estrategias del mercado juvenil. ${ }^{29}$

También la simplificación de los integrados, es decir, intérpretes benévolos y hasta inocentes porque consideran que las redes sociales no son un problema educativo específico y singular. Para los integrados, las redes son un problema educativo más, entre otros muchos. No consideran que el nuevo horizonte espitemológico que están abriendo pueda ser determinante en las nuevas configuraciones de la sensibilidad, el conocimiento y la acción de las futuras generaciones. Por ello consideran que no requiere un tratamiento singularizado ni especializado. Como todos los procesos de innovación cultural, los apocalípticos y los integrados focalizan dos grupos de actitudes que nos pueden ayudar en los procesos de deliberación organizativa. No se trata sólo de buscar simplemente un justo medio en cada situación sino de facilitar la deliberación en los espacios públicos educativos para que la organización se comprometa con el dinamismo de la responsabilidad.

Como señala el reciente informe de la Fundación Encuentro, con independencia de los riesgos y las oportunidades que presentan las redes sociales, todos los expertos insisten en una idea clave: "se trata de una cuestión necesitada de un enfoque conjunto entre la familia, el colegio y la misma sociedad civil" (subrayado nuestro) ${ }^{30}$. Una sociedad civil donde las organizaciones juveniles deberían

\footnotetext{
29 Umberto Eco identificó con las categorías apocalípticos/integrados dos actitudes básicas ante los fenómenos culturales cuando analizó la cultura de masas que en algunos círculos académicos se consideraba enfrentada a la cultura democrática. El debate sigue vivo y la lectura del libro de Eco es oportuna, sobre todo cuando los educadores nos enfrentamos a fenómenos culturales nuevos y de carácter masivo, cfr. (1965) (varias eds.) Apocalípticos e Integrados, Barcelona, Lumen.

30 Informe España 2010, op. cit., p. 154.
} 
tomar una clara posición de liderazgo y denunciar los reduccionismos que se hacen desde la economía más utilitarista (que identifica sociedad civil sólo con ámbito económico no regulado por las administraciones públicas) o desde la teoría política más simplificadora (que identifica la sociedad civil con el ámbito artístico, deportivo, decorativo o cultural en el más simple de los sentidos). Como hemos señalado en otros trabajos, está reinvención cultural y ética de la sociedad civil es determinante para superar no sólo la crisis económica sino una crisis de civilización que a veces parece no confiar en sí misma. ${ }^{31}$

\subsection{Rumbo e identidad organizativa}

El segundo desafío está relacionado con el rumbo de su navegación, es decir, la clarificación de las metas que persiguen y la delimitación de lo que técnicamente llamamos misión-visión. ¿̇Hacia dónde vamos? ¿ Tenemos claro el rumbo marcado para acercarnos al destino que nos hemos propuesto? Aunque los estatutos incluyan la respuesta, el grupo tiene que actualizarlas de manera permanente para que su identidad sea viva y significativa. Son preguntas que los grupos deben hacerse periódicamente, lo que no significa que se realicen insistentemente de forma neurótica en cada asamblea porque generarían desmoralización y desánimo.

Aunque en la vida de una organización juvenil sea necesaria su identidad estatutaria resulta insuficiente para mantener viva la organización. Por eso, cuando hablamos de organizaciones juveniles vivas hablamos de identidades narrativas, es decir, compartidas, reelaboradas y resignificadas diariamente por sus miembros. La mayor parte de las organizaciones juveniles no son conscientes de que están obligadas a replantearse la identidad en tiempos de globalización y de redes sociales donde la educación para el tiempo libre significa algo diferente a lo que significaba en el siglo pasado.

Nos encontramos ante un momento crítico para las organizaciones juveniles, que han pasado tres fases. En la primera, las organizaciones juveniles tenían como finalidad completar la educación que la familia y la escuela no realizaban, su función era facilitar la integración social y la educación integral para que los jóvenes evitaran la calle, la desocupación y el tiempo de ocio como un tiempo sin orden o fuera de cierto orden moral. Las organizaciones juveniles de confesiones religiosas, de agrupaciones políticas, o de vocación filantrópica internacionalista

${ }^{31}$ Sobre este concepto de sociedad civil puede verse nuestro trabajo (2001) Ética y Voluntariado. Una sociedad sin fronteras, Madrid, PPC, $2^{\mathrm{a}}$ edición. 
que surgieron antes de la segunda guerra mundial tenían esta finalidad: facilitar la coherencia en la socialización secundaria de los jóvenes ${ }^{32}$.

En una segunda fase, las organizaciones cuestionan la educación que proporcionan la familia y la escuela. Bajo el impacto de planteamientos culturales alternativos y movilizados por el liderazgo de las tres M (Marx, Mao y Marcuse) se denuncia la moral cerrada de las instituciones tradicionales como la familia y la escuela; reclaman una moral abierta entendida en términos nietzscheanos como una moral "sin obligación ni sanción" 33 . Se plantea una profunda crisis de las organizaciones porque en lugar de facilitar la integración social o la coherencia para tender puentes entre familia-escuela, lo que hacen es cuestionar la coherencia, es decir, el valor de la integración-cohesión social y de la educación formal (familia y escuela). La educación no formal se identifica con propuestas de cambio e innovación social de carácter alternativo. En lugar de promover la continuidad con las tradiciones educativas de la escuela o la familia se promueve la discontinuidad con la tradición. Una discontinuidad que se traslada al interior de la vida familiar y la vida educativa despreciando los procesos de formalización e institucionalización.

Con la llegada de la televisión y el progresivo desarrollo de las redes sociales comienza una tercera fase. No sólo por el hecho de que la globalización rompe fronteras y muros sino porque aparecen "pantallas" que condicionan los procesos de socialización anteriores. La relación de los adolescentes con el televisor, las videoconsolas, los ordenadores o el móvil transforma la organización cognitiva de las relaciones con uno mismo, con los próximos o lejanos y, por lo que aquí nos ocupa, con las instancias socializadoras tradicionales (familia, escuela). Emerge así una generación tecnológicamente equipada, instrumentalmente precoz, más autónoma y multifuncional pero desconcertada ante los nuevos riesgos ${ }^{34}$.

\footnotetext{
32 Utilizaré el término coherencia para describir lo que con distintos términos y desde distintas tradiciones de psicología de la personalidad llamamos una personalidad equilibrada y madura. No tiene el sentido epistemológico de sistema cerrado sino un sentido ético o más propio de la razón práctica como ajustamiento, armonización y equilibrio reflexivo entre proyectos/realizaciones, ideas/creencias, experiencias/expectativas, decires/haceres, valores finalistas/valores instrumentales, fines/medios.

33 Utilizo los términos moral cerrada y moral abierta en el sentido que H. BERGSON les otorga, (2008) Les deux sources de la morale et de la religion, París, PUF, nouvelle edition critique, cfr. (1996) Las dos fuentes de la moral y la religión, Madrid, Tecnos.

${ }^{34}$ Sobre los nuevos riesgos de esta, cfr. La generación Interactiva, op. cit., p. 547.
} 
Ahora las organizaciones juveniles tienen desafíos de socialización nuevos: atrás quedaron los tiempos de facilitar o cuestionar la coherencia entre familia-escuela-tiempo libre. Sin demasiadas ambiciones organizativas, las organizaciones necesitan descubrir la coherencia, es decir, la necesidad de evitar patologías, neurosis y disonancia cognitiva entre instancias socializadoras que gestionan conocimientos sin plantearse la necesidad de coordinación, sistematización o memorización. El desconcierto y hasta la impotencia de la familia o la escuela proporcionan a las organizaciones competencias nuevas.

\subsection{Mediación innovadora y constructiva}

Una de las competencias más interesantes que ahora tienen las organizaciones juveniles podría llamarse mediación innovadora y constructiva. Cuando la comunicación entre la familia y la escuela no es fluida, cuando la comunicación entre la vida familiar y la vida cívica es inexistente o despreciada por unos u otros, cuando la escuela degenera en academicismo porque no se plantea problemas de excelencia, exclusión, marginación o cohesión social, entonces constatamos que las conexiones familia-escuela-calle (sociedad) si no están rotas al menos están lesionadas o fracturadas.

No debemos confundir la mediación con la inter-mediación, como si las organizaciones juveniles debieran terciar en los conflictos y convertirse en agentes de inter-mediación o negociación. Tampoco la mediación con la negociación o el arbitraje entre instancias socializadoras. Me refiero a la mediación como tercer espacio, es decir, espacio nuevo de comunicación, ámbito diferente a la familia, la escuela o la calle donde se pueden reconstruir hábitos de socialización y procesos cognitivos-emocionales en un contexto organizativo nuevo. Sin ser actividades familiares o escolares, las actividades que realizan algunos grupos juveniles como acampadas, convivencias, marchas o incluso visitas a determinados centros para realizar actividades de voluntariado tienen un potencial cívico-formativo que no puede ser despreciado. El horizonte educativo del tercer maestro se construye a partir de este ámbito de mediación ${ }^{35}$.

${ }^{35}$ Cfr. J. F. SIx (2001) Dinámicas de mediación, Barcelona, Paidós, trad. J. Giró. Este concepto de mediación es central en las dinámicas de justicia juvenil y resolución de conflictos, como ha señalado el profesor GIRó en (2001) "La justicia y la mediación": Educación Social 8, pp. 215-235. Se trata de un concepto hermenéutico y personalista de mediación que podemos encontrar en los trabajos de $P$. RICOEUR (2008) Lo justo II, Madrid, Trotta, trad. Tomás y Agustín Domingo. 


\subsection{Personalización e Interiorización}

Las organizaciones juveniles pueden facilitar los procesos de personalización e interiorización que son fundamentales en la adolescencia. A pesar de que el propio nombre de las redes sociales parece implicar un incremento de la sensibilidad social y comunitaria de los adolescentes, en realidad se trata de una gestión nueva del individualismo cultural. La relación del adolescente con la pantalla siempre es a través de "su cuenta". Aunque sean importantes los grupos y las comunidades que se crean en la red, la relación del individuo con la pantalla siempre es individual reclamando atención a lo que sucede o aparece en la pantalla en detrimento de lo que sucede o aparece en el entorno físico. Las redes no sólo aproximan sino que también alejan, las redes no sólo facilitan información sino distracción. En los procesos de personalización que facilita la red el prójimo aunque sea real no está presente, aunque esté informativamente cerca es inofensivo para la movilidad cotidiana.

La personalización a la que nos referimos tiene como finalidad invitar a las organizaciones juveniles a que realicen actividades que faciliten usos personalizados y controlados de las redes. Frente al uso despersonalizador, anónimo e incontrolado de las redes, es importante mostrar que también hay un uso personalizador que no sólo está relacionado con la escuela, la familia o el trabajo sino con el tiempo libre. No para utilizar el tiempo libre delante de las pantallas e incentivar los procesos de ensimismamiento moral, sino para descubrir en el tiempo libre dimensiones físicas y reales de un prójimo que no es virtual ${ }^{36}$. Los estudios sobre la nueva generación como nueva generación interactiva y multifuncional no sólo conceden mayor valor a la autonomía del menor ante la red sino que reconocen la precocidad, los riesgos y la vulnerabilidad e esta autonomía. Son menores autodidactas en la incorporación de Internet a sus vidas que están generalizando lo que los expertos han llamado "cultura del dormitorio" ${ }^{37}$.

No es menos importante el desafío de la interiorización en unas redes sociales que proporcionan información pero que no nos garantizan conocimiento y criterio. La interiorización no es ensimismamiento sino un camino único y personal para

\footnotetext{
${ }^{36}$ Es importante señalar que la educación en las redes sociales no puede reducirse a la gestión o administración de la conexión sino a la necesidad y valor de la desconexión. En los procesos educativos de personalización la desconexión es tan importante como en los procesos de interiorización el silencio, véase nuestro artículo El valor educativo del silencio, $A B C, 21 / 12 / 2006$.
}

${ }^{37}$ X. Bringué, Ch. SÁDABA y J. TolsÁ, La generación Interactiva, op. cit., p. 550. 
el autoconocimiento, el crecimiento y la maduración. Las redes sociales fuerzan a que los menores tengan que identificarse, clarificar sus gustos, preferencia y valores. Se trata de un hecho solo aparentemente positivo puesto que estamos ante una identidad precoz. Al fijar gustos, jerarquizar preferencia y mostrar valores, el perfil del menor se reifica, es decir, pierde parte de su dinamismo y-probablemente- hipoteca el proceso de maduración.

Las redes sociales tienen una función engolosinadora porque son capaces de inquietar la personalidad, despertar nuevos deseos y movilizar al yo en algo que lo dispersa y que lo mantiene atrapado ${ }^{38}$. Aunque faciliten información y posibilidades en el ámbito informativo, las redes están organizadas para "pescar" al usuario $y$, si es menor, aprovechan la vulnerabilidad de su personalidad. Aunque las redes pueden atrapar en todas las edades, la precocidad que se está produciendo en el acceso y la vulnerabilidad de los menores exigen atención educativa especial.

Las organizaciones juveniles pueden entrenar para la desconexión y el desenredo 0 , dicho con otras palabras, tienen que contribuir a que las redes faciliten el ejercicio de la responsabilidad de los menores. Los fracasos escolares y familiares de esta edad están relacionados con la falta de entrenamiento en la desconexión ${ }^{39}$. Las pantallas han sustituido a los libros y cuando la lectura no se realiza en pantalla es más incómoda y aburrida. El atractivo de las redes ayuda a realizar múltiples tareas a la vez y supone un reto para la vida familiar porque nos encontramos ante el reto de una familia interactiva.

El atractivo de las redes, como constatan los redactores del informe de la Fundación Encuentro, "potencia elementos que afectan (junto a otros) a los estilos cognitivos y emocionales de los adolescentes de hoy, como la dificultad para la síntesis y la abstracción, el predominio de la imagen, de lo visual y lo concreto en las formas de abordar y asumir las realidades. Parcelan la percepción de las cosas, les falta atención y capacidad de concentración..." 40 .

\footnotetext{
${ }^{38}$ Según la XXII edición del diccionario de la RAE, el verbo "engolosinar" tiene dos significados, relevantes para nuestro trabajo: "excitar el deseo de alguien con algún atractivo" y "aficionarse, tomar gusto a algo".

${ }^{39}$ Dentro de la vida familiar se observa una diferencia importante entre el padre y la madre, el primero suele ser una referencia para la generación interactiva y la segunda es una referencia para lo que llaman "offline", Cfr. BRINGUÉ y otros, op. cit., p. 555.

${ }^{40}$ Op. cit., p. 124.
} 


\subsection{Empatía y reconocimiento}

El último desafío que planteamos está relacionado con la empatía y el reconocimiento. Los diferentes diagnósticos e investigaciones sobre la convivencia escolar siempre indican que la mejor terapia para promover la convivencia es la empatía. En esta tercera fase histórica en la que hemos situado a las organizaciones juveniles, el desafío de la empatía no se limita a compartir estados de ánimo o incrementar la sensibilidad cívica y comunitaria de los adolescentes. El desafío es mucho más básico y estructural porque en otras fases históricas de la vida organizativa, el menor no tenía que ser capacitado para la empatía porque la familia y la escuela ya los capacitaban, habían despertado en ellos este germen de comunidad o semilla del nosotros que describimos como empatía.

Cuando hablábamos de la empatía nos referíamos a los estados de ánimo de las personas y la definíamos como "identificación mental y afectiva con el estado de ánimo de otro". Dos individuos diferentes puede compartir un mismo estado de ánimo, dos personas diferentes y diferenciadas pueden llegar a compartir un mismo estado de ánimo. Ahora, en la nueva edición del diccionario de la lengua, la empatía aparece definida como "capacidad para identificarse con otro y compartir sus sentimientos". La educación para la empatía no es sólo la educación para el reconocimiento de una identidad común sino para compartir determinados sentimientos.

Estamos ante un desafío para despertar la sensibilidad comunitaria, para desarrollar la capacidad de identificarse con otro y para compartir con los demás. Esto no significa que las organizaciones juveniles tengan que ser organizaciones gregarias, sectarias o cerradas, significa que las organizaciones juveniles deben conocer sus nuevas responsabilidades. En sociedades liberales, el desafío de la empatía y el reconocimiento se ha convertido en una tarea explícita, formal y prioritaria porque el yo no sólo aparece aislado, sino blindado. La clave de la construcción de un nosotros no está sólo la orientación de ese nosotros en una determinada dirección histórica sino en el descubrimiento de un nosotros posibilitador.

La identificación con el otro es un proceso cada vez más complejo cuando las redes sociales en lugar de liberarnos terminan atrapándonos, por eso cada vez son más necesarios los espacios de mediación educativa que pueden generar organizaciones de tiempo libre. Es importante recordar que el desafío de la empatía no es únicamente la capacitación para identificarnos con el otro y compartir sus sentimientos. La empatía no sólo es necesaria para reconocer al otro en el sentido de 
acordarnos de él e identificarlo como prójimo; es necesaria para tener presente que reconocer también es agradecer. Por eso una de las tareas más olvidadas (y no por ello menos importantes) en el horizonte educativo del tercer maestro sigue siendo la de educar para el agradecimiento.

\section{Conclusiones}

Al terminar este recorrido por los desafíos que las redes sociales plantean a la educación no formal, ha llegado el momento de recapitular las ideas básicas de nuestra reflexión. En primer lugar, la constatación de que las teorías sobre la educación democrática reconocen el gran territorio educativo que tenemos pendiente en el tiempo libre y la educación informal. Para alinear los valores finalistas con los valores instrumentales de los menores necesitamos completar los magisterios tradicionales de la familia y la escuela con magisterios innovadores; que no sean subsidiarios de los procesos de escolarización, sino complementarios. Además del primer maestro (familia) y el segundo (escuela), podemos pensar la calle y el ocio como espacios que están exigiendo la emergencia del horizonte educativo del tercer maestro (educación no formal). Aquí se sitúan los desafíos de las redes sociales y las TICS.

No sólo como herramienta o instrumento educativo sino espacio comunicativo desde el que se configura el complejo imaginario social y cultural de los menores. El nuevo escenario educativo ante el que nos sitúan las redes sociales exige que no limitemos nuestras propuestas de educación cívica en términos de valores ${ }^{41}$. Este potencial normativo de los valores para construir una ciudadanía activa no exige el olvido o la marginación del potencial educativo de las virtudes (o actitudes, carácter y ejemplos) para promover una formación integral de la persona. El escenario de una ciudadanía digital al que se enfrentan los programas de ciudadanía democrática es una buena oportunidad para revisar estrategias de educación moral que incluyan una configuración integral de la persona, es decir, una visión no sólo cognitivo-racional sino emocional y cordial.

\footnotetext{
${ }^{41}$ La educación cívica en general y la educación para la ciudadanía activa en particular no puede plantearse de espaldas al potencial ético, epistemológico y axiológico de las religiones. Sobre este tema puede verse nuestro trabajo (2011) Ciudadanía activa y religión. Las fuentes pre-políticas de la ética democrática, Madrid, Encuentro.
} 
Con este horizonte de la formación integral emergen nuevas posibilidades para que la educación democrática también alcance espacios de socialización que no sean la escuela y la familia. Espacios que hoy están determinados por la presencia de las redes sociales en la re-configuración del imaginario juvenil y la transformación radical de las pautas de socialización. Espacios con algunos desafíos organizativos que nos hemos atrevido a señalar (identidad, personalización, empatía, etc.). Espacios llenos de interrogantes para los profesionales con firmes convicciones educativas $y$, a su vez, con nuevas responsabilidades para los profesionales de la mercadotecnia y la comunicación pública. Espacios donde el ocio y el tiempo libre seguirán siendo, como vieron las organizaciones juveniles más proféticas, horizones de terceros maestros para continuar la arriesgada aventura de educar. ${ }^{42}$

${ }^{42}$ Agradezco a los profesores Juan Escámez, Raúl Espejo, Zoraida Mendiwelso, Amparo Mir, Adolfo Montalvo, Inmaculada Ros, Leopoldo Salinas, José Ignacio Tudela y M. Victoria Vergara los comentarios y sugerencias en la versión final de este trabajo. 